\title{
BREVE HISTORIA DEL PRECEDENTE EN INGLATERRA
}

\section{A SHORT HISTORY OF PRECEDENT IN ENGLAND}

\author{
Víctor Saucedo \\ Universidad Autónoma de Madrid
}

SUMARIO: I. LA DOCTRINA DEL PRECEDENTE EN LA ACTUALIDAD; II. EL PRECEDENTE MEDIEVAL; III. EL PRECEDENTE EN LA ÉPOCA MODERNA; IV. LA DOCTRINA DEL PRECEDENTE EN EL SIGLO XIX; V. PROSPECTIVAS DE ESTUDIO

Resumen: En este artículo apuntaré una posible línea de investigación sobre la historia y teoría del precedente vinculante, uno de los aspectos centrales del rule of law en países de la familia angloamericana. Entre otras cosas describiré cómo, aunque esta doctrina tiene unos orígenes relativamente recientes, la idea de precedente puede remontarse a la propia formación del common law. También hablaré brevemente de la aparición de la moderna doctrina del precedente y qué relación guarda con el desarrollo medieval anterior. Este marco me servirá para encuadrar y esbozar qué posible línea de investigación se pueden abrir.

Abstract: In this article, I will propose a line of research about the theory and history of the doctrine of precedent, one of the crucial elements of the rule of law of the Anglo-American legal family. Among other things, I will show how the idea of precedent goes back to the formative period of the common law. I will also discuss the emergence of the modern doctrine of precedent as well as how is related to its medieval development. Upon this framework, I will discuss my proposed line of research.

Palabras clave: Common law, doctrina del precedente, historia jurídica, historia del derecho, período moderno, edad media, siglo diecinueve, ratio decidendi, Year Books, obiter dictum, law reports, Reino Unido, Inglaterra, razonamiento jurídico.

Keywords: Common law, doctrine of precedent, legal history, Early Modern period, Middle Ages, nineteenth century, ratio decidendi, Year Books, obiter dictum, law reports, United Kingdom, England, legal reasoning.

\section{LA DOCTRINA DEL PRECEDENTE EN LA ACTUALIDAD}

Para empezar, debemos clarificar qué se entiende por precedente y cuáles son los parámetros de la doctrina del precedente vinculante en Inglaterra y Gales en la actualidad. En general, el término precedente conlleva un significado normativo y temporal. Por un lado, el término alude a una pauta de conducta pasada en comparación con una situación presente a la que precede. De aquí el uso del término precedente, esto es, aquello que precede. Por otro lado, el término 
indica que esa pauta de conducta pasada puede servir como modelo o ejemplo para una conducta futura. Por lo tanto, en un contexto jurídico, el término precedente se refiere a un caso pasado que puede servir de modelo para decidir casos futuros. Ahora bien, no cualquier caso puede servir de modelo a un caso subsiguiente, sino aquel que se asemeje en hechos y derecho al caso para el que se pretende como modelo o ejemplo. ${ }^{1}$

Pasemos ahora a ver cuáles son los aspectos más relevantes de la doctrina del precedente. Para empezar, el precedente se entiende como vinculante. Ello quiere decir que el precedente mismo tiene fuerza de obligar y no es simplemente la ilustración o ampliación de una norma externa. Esto es, en contraste con otras tradiciones, no debemos entender el precedente como interpretación o ampliación del código que sería la norma con fuerza de obligar. El precedente en sí mismo es fuente de derecho. ${ }^{2}$

A continuación, en segundo lugar, tenemos la jerarquía de tribunales. Uno de los aspectos fundamentales de la doctrina del precedente es que el tribunal superior en la jerarquía vincula al tribunal inferior en la jerarquía. Tribunales que se encuentran al mismo nivel en la jerarquía o de jurisdicción coordinada están vinculados por sus propias decisiones pasadas con excepción del tribunal de mayor rango en la jerarquía (en la actualidad se trata del Tribunal Supremo, anteriormente la Cámara de los Lores). Las decisiones de los tribunales inferiores tienen un carácter persuasivo pero no vinculante sobre las decisiones de tribunales superiores. Se sigue, por lo tanto, que en la práctica son los tribunales superiores (Tribunal de Apelación, Tribunal Supremo) los que determinan el curso de los precedentes. ${ }^{3}$

Un tercer elemento es el respeto que se debe a la decisión particular. En otras palabras, a diferencia de otras jurisdicciones, en la tradición de common law, no es la tendencia de una línea de decisiones en un sentido la que constituye el precedente. Antes al contrario, si un tribunal encuentra una sola decisión que es lo bastante parecida en hecho o derecho, entonces está obligado a seguir esa decisión con independencia de que esté avalada por otras decisiones en el mismo sentido. ${ }^{4}$

Dicho todo esto, se nos presenta la duda de qué es exactamente aquello que vincula de un precedente. En un caso podemos encontrar múltiples elementos: los argumentos de las partes, las diferentes opiniones de los miembros del tribunal en el supuesto de órganos colegiados, así como las diferentes líneas argumentales ensayadas por parte del juez al decidir el caso. De entre todos esos elementos, debemos distinguir aquella resolución, principio o norma por la que el caso se nos presenta como una autoridad, como un precedente vinculante. Esta parte es la que se denomina como la ratio decidendi, la razón para decidir. Aquí

\footnotetext{
1 N. DUXBURY, The Nature and Authority of Precedent, Cambridge University Press, Cambridge, 2008, pp. 1-14.

2 R. CROSS y H. J. W. , Precedent in English Law. $4^{\mathrm{a}}$ ed., Oxford University Press, Oxford; New York, 1991, 3-5., LQ Rev., vol. 50 (1934), p. 41.

3 R. CROSS y H. J. W. , Precedent in English Law, op. cit. pp. 5-6, 8-9, 11-13, 24-25. A. L. GOODHART, "Precedent in English and Continental Law", L. Q. Rev., vol. 50, 1934, pp. 41-42.

4 R. CROSS y H. J. W. , Precedent in English Law, op. cit. pp. 5, 7-8, 11, 20. A. L. GOODHART, "Precedent in English and Continental Law", op. cit., p. 44.
} 
quiero señalar que, aunque uno se siente tentado de usar nuestro término motivación, prefiero conservar esta expresión. A diferencia de motivación, esta expresión está ligada a otra con la que precisamente se designa todo aquello que no es razón para decidir pero que puede tener un valor de autoridad persuasiva en casos parecidos. Esto es, la expresión obiter dicta, lo que se ha dicho a propósito de o en el decurso de. En otras palabras, en la tradición de common law la razón para decidir no siempre aparece determinada de manera clara e inequívoca, por lo que con frecuencia es necesaria una labor de interpretación para establecerla. De hecho, se puede decir que en el fondo la formación del estudiante de common law consiste en el aprendizaje del arte de encontrar la ratio decidendi y separarla de los obiter dicta. ${ }^{5}$

La distinción ratio decidendi / obiter dicta era el cuarto aspecto de la doctrina del precedente que quería señalar. Un quinto aspecto que también me gustaria comentar es el de la función que se supone cumple esta doctrina en el seno del common law. El hecho de que los jueces tengan que decidir en armonía con decisiones anteriores conlleva coherencia, uniformidad y certeza tanto en dichas decisiones como en el proceso de producción normativa a través de la actividad judicial. En otras palabras, con esta doctrina del precedente vinculante se evita que las decisiones aparezcan como arbitrarias puesto que los jueces deben dar razones y las mismas deben aplicarse en vista de las decisiones pasadas. Al mismo tiempo, se asegura que el derecho crezca por la vía jurisprudencial en coherencia con el resto del ordenamiento. ${ }^{6}$

En ese sentido, se suele apuntar que la coherencia, uniformidad y certeza que genera la doctrina del precedente vinculante deriva en el fondo de la jerarquía de los tribunales. Que los tribunales inferiores estén obligados a seguir las decisiones análogas de los superiores garantiza precisamente que pueda existir la propia idea del precedente vinculante. De otra manera, nos encontrariamos con el problema de decisiones incompatibles. Por ejemplo, en el caso de que un tribunal se encontrara ante dos precedentes contradictorios, uno originado en una jurisdicción inferior y el otro en una superior. Lo mismo se puede decir del principio según el cual los tribunales están vinculados por sus propias decisiones (con excepción del Tribunal Supremo, pero esto es un desarrollo reciente del 1966). En el momento en que no existiera tal jerarquía, la distinción entre precedentes vinculantes y aquellos solamente persuasivos por pertenecer a una jurisdicción inferior desaparecería, y con ella la posibilidad de establecer con certeza el derecho.

Sin embargo, la aplicación estricta del principio de la jerarquía de los tribunales implica que se deben seguir incluso aquellos precedentes en los que los jueces aprecian que el principio en el que se basa es erróneo, o cuando la aplicación de este al caso presente pueda conllevar que se produzca una decisión injusta. En estos casos sería deseable que el principio de la jerarquía de los tribunales se relajara, al menos cuando existiera una buena razón para hacerlo. Esto permitiria, por otro lado, que el common law pueda desarrollarse de manera

\footnotetext{
5 R. CROSS y H. J. W. , Precedent in English Law, op. cit. pp. 6, 13-15, 18, 39-96. A. L. GOODHART, "Determining the Ratio Decidendi of a Case", The Yale Law Journal, vol. 40, num. 2, 1930, pp. 161-183.

${ }^{6}$ R. CROSS y H. J. W. , Precedent in English Law, op. cit. pp. 11-12, 103-104. A. L. GOODHART, "Precedent in English and Continental Law", op. cit., pp. 58-65.
} 
flexible. En otras palabras, una aplicación estricta de este principio de la jerarquía implica una excesiva rigidez en el ordenamiento, impidiendo que las normas se puedan adaptar a los cambios que se den en la sociedad.

El problema de si los tribunales, particularmente aquellos de rango superior en la jerarquía, pueden apartarse de la doctrina del presente vinculante en determinadas circunstancias ha sido uno de los ejes del debate doctrinal del siglo $\mathrm{XX}$. No este el momento de entrar en los vericuetos del mismo. Baste decir que desde finales del siglo XIX y hasta mediados del XX esta doctrina se aplicó de manera estricta y que desde 1966, cuando la Cámara de los Lores emanó el documento titulado The Practice Statement, se considera que el vértice de la jerarquía, esto es, el Tribunal Supremo, no está vinculado por sus decisiones anteriores, con lo cual puede apartarse de ellas siempre y cuando esté justificado porque el precedente conlleve error o injusticia. ${ }^{7}$

Se sigue de lo que acabo de mencionar que algunos aspectos de la doctrina del precedente vinculante son relativamente recientes. De hecho, en conjunto, la moderna doctrina del precedente vinculante no empieza a vislumbrarse hasta finales del siglo XVIII y realmente no cobra la forma que tiene hoy en día hasta mediados del siglo XIX. Con todo, la idea de precedente es casi tan antigua como el propio common law, y la doctrina moderna surge del fértil suelo de los siglos XVI y XVII cuando ciertas circunstancias permitieron un cambio en la actitud hacia el precedente. En ese sentido, la historiografia del precedente se ha preguntado por qué esta doctrina no se formuló antes y cuáles fueron los factores que posibilitaron su aparición. Veamos qué respuestas se han dado.

\section{EL PRECEDENTE MEDIEVAL}

La referencia a precedentes es casi contemporánea a los orígenes del sistema jurídico de common law. Sin embargo, el uso que se hacia en la edad media del precedente distaba de la idea contemporánea. Por ejemplo, el libro "De Legibus Et Consuetudinibus Angliæe" atribuido a Bracton (que es una exposición de las leyes y costumbres de Inglaterra escrito entre 1230 y 1260, y probablemente destinado a la enseñanza jurídica) inaugura la tradición de citar precedentes. En él hay numerosas referencias a unos quinientos casos tomados de diversos rolls de los tribunales centrales (Common Pleas, King's Bench y justicia itinerante o Eyres) que indican que se les concedian algún valor como precedentes. Ahora bien, el tratamiento que recibian no era el de precedentes vinculantes. Puesto que el propósito de Bracton es pedagógico, los precedentes sirven más bien como ilustraciones de cuestiones de derecho para mostrar a jueces y abogados de qué manera y en qué modo se deciden los pleitos. Pero en absoluto se concibe que estos casos puedan ser citados en un tribunal aunque sí tengan un valor testimonial, como prueba o evidencia de ciertos principios y normas jurídicas. Los precedentes en Bracton tienen la función o el valor de mostrar cuál es el derecho, pero no son en ninguna medida vinculantes, sino en todo caso persuasivos. ${ }^{8}$

\footnotetext{
7 R. CROSS y H. J. W. , Precedent in English Law, op. cit. pp. 97-164.

8 J. H. BAKER, An Introduction to English Legal History, $4^{\mathrm{a}}$ ed., Ohio Butterworths, LexisNexis, Londres, Dayton (Ohio), 2002, pp. 196-197. T. E. LEWIS, “The History of Judicial Precedent. I", L.
} 
Los tribunales podían en ciertas ocasiones seguir y distinguir precedentes, como prueba de cuál era el derecho aplicable, pero no porque se sintiesen vinculados por decisiones previas. Esto era asi, entre otras razones, porque las actas del proceso (la sede material, los rolls) no recogian más que la sentencia sin incluir ni las razones para decidir, ni los precedentes citados, si los habia; la propia estructura y naturaleza del proceso medieval impedía que tales cuestiones se reflejaran documentalmente cuando se discutian en sede judicial. Quizá sea necesario en este punto realizar un breve excurso para describir los aspectos del proceso medieval que son relevantes a la hora de explicar por qué, aunque en la edad media existiera la idea de precedente, éste nunca se consideró vinculante. ${ }^{9}$

La primera cosa en la que debemos detenernos es la aparición del juicio con jurado a mediados del siglo XII. Esto supuso un cambio drástico en las formas procesales. Modos de prueba como la ordalia y la compurgación que habían dominado el proceso de la justicia comunal iban a ser desplazados por el veredicto del jurado, convertido en eje del proceso de los tribunales regios. ${ }^{10}$ También hemos de considerar la expansión de la jurisdicción regia a través del instrumento procesal del writ, que era una carta emanada de la chancillería regía a petición de parte y dirigida al sheriff (el oficial del rey en el condado) con orden de requirir la comparecencia del demandado ante los jueces regios para responder la demanda. Con este instrumento, por tanto, se ganaba el acceso a dicha jurisdicción y se daba comienzo al proceso. Writ y juicio del jurado suponian que la discusión jurídica entre las partes acontecía justo antes del juicio, cuando comparecian en audiencia pública ante el tribunal de Westminster para celebrar el contencioso oral conocido como pleading (que quizá sería demasiado reductivo traducir como alegaciones). ${ }^{11}$

El pleading da comienzo con una narración de los hechos por parte del demandante o plaintiff que amplía el contenido enunciado de manera formal en el writ. Una vez terminado, el demandado o defendant tiene la oportunidad de contestar. Aquí se presentan dos posibilidades. Puede por un lado demur, esto es, admitir los hechos y objetar la suficiencia (o validez) del writ, alegando que los hechos declarados no son causa de acción. Esta respuesta del demandado genera una cuestión de derecho cuya resolución atañe al tribunal y que, por lo tanto, potencialmente contribuye al establecimiento de derecho substantivo. Esta opción, sin embargo, conlleva notables riesgos para la parte demandada, puesto que se admiten los hechos en cuestión y, por tanto, se ciega la posibilidad de que haya juicio de los mismos. Por otro lado, el demandado puede simplemente negar los hechos, o una parte de ellos (traverse), lo cual generará una cuestión de hecho cuya respuesta atañe al jurado. Precisamente, esta etapa procesal del pleading

Q. Rev., vol. 46, 1930, pp. 209-212. T. F. T. PLUCKNETT, A Concise History of the Common law, $5^{\text {a }}$ ed., Little, Brown and Co., Boston, 1956, pp. 342-345.

9 J. H. BAKER, An Introduction to English Legal History, op. cit., pp. 197-198. N. DUXBURY, The Nature and Authority of Precedent, op. cit., p. 32.

10 J. H. BAKER, An Introduction to English Legal History, op. cit., pp. 72-73. T. F. T. PLUCKNETT, A Concise History of the Common law, op. cit., pp. 106-138.

11 J. H. BAKER, An Introduction to English Legal History, op. cit., pp. 53-57. T. F. T. PLUCKNETT, A Concise History of the Common law, op. cit., pp. 354-360. 
tiene como propósito formular una cuestión sobre los hechos del caso que el jurado ha de determinar. ${ }^{12}$

En la práctica, puesto que esta fase procesal era oral y su resultado no se recogia en el acta hasta que el oficial del tribunal lo incluia en un registro escrito (roll), era frecuente que se produjera lo que se ha llamado el pleading tentativo. En otras palabras, los demandantes avanzaban sus argumentos sólo de manera provisional, para comprobar cuál era la reacción del tribunal. Si una objeción era mirada con reticencias se retiraba antes de que pasara al acta y se probaba otra nueva, o se procedía a negar los hechos generando una cuestión fáctica que por tanto pasaría a la fase probatoria. Si la objeción se consideraba potencialmente válida, el demandante probablemente procedia a ofrecer una nueva versión de los hechos que pudiera dar lugar a una cuestión fáctica.

Este modo de proceder era particularmente favorecido por los jueces, en esta época bastante reticentes a pronunciarse sobre cuestiones de derecho. Preferian dejar las decisiones en manos del jurado para luego, sobre el veredicto, proceder de manera automática dictando sentencia. En este intercambio provisional entre las partes el juez no jugaba un papel resolutorio, sino más bien actuaba como un árbitro que reconducia las partes hacia una cuestión de hecho que pudiera ser respondida por el jurado. Se sigue así que la discusión era de naturaleza hipotética puesto que nunca versaba sobre hechos afirmados sino presupuestos. Por lo tanto, los hechos serian determinados más tarde en el juicio y, habiendo esclarecido las cuestiones de derecho en el pleading, los jueces no se sentían obligados a fundamentar las razones en las que basaban sus sentencias. Igualmente, dichos problemas jurídicos y los argumentos realizados no se recogian formalmente puesto que se resolvian de manera tentativa en el pleading oral. De todo ello se sigue que no existian las condiciones para que las decisiones judiciales se constituyeran en precedentes. ${ }^{13}$

Esta estructura del proceso se aprecia precisamente en los primeros law reports de la historia jurídica inglesa, los llamados Year Books. Éstos comenzaron a producirse y circular en forma manuscrita en el siglo XIII y su uso se extendió hasta el siglo XVI. En cierto sentido se puede considerar que son los antecedentes de los modernos law reports, pero su contenido y su naturaleza eran bastante diferentes. Los Year Books, como he indicado, se centran en esa parte del proceso que quedaba fuera de las actas, el pleading. A sus redactores no les interesaba precisar cómo se habian resuelto las cuestiones de derecho surgidas a propósito de un caso. En realidad, es bastante frecuente encontrar que los casos recogidos en los Year Books reflejan puntos que nada tienen que ver con la cuestión final, o incluso que esta misma sea omitida. Los autores de estos primitivos law reports se centraban en la discusión anterior a la cuestión final. Esto es, les interesaba los argumentos que las partes y los jueces desplegaban en el curso del pleading tentativo más que la decisión acerca de los mismos. Por eso los Year Books tienen una forma dialógica en la que no es raro que las decisiones formales de los jueces aparezcan como un argumento más.

\footnotetext{
12 J. H. BAKER, An Introduction to English Legal History, op. cit., pp. 76-78. T. F. T. PLUCKNETT, A Concise History of the Common law, op. cit., pp. 399-401, 409-410, 413-415.

13 J. H. BAKER, An Introduction to English Legal History, op. cit., pp. 78-81. T. F. T. PLUCKNETT, A Concise History of the Common law, op. cit., pp. 401-404.
} 
El propósito de los Year Books no era recoger las rationes decidendi expresadas por un juez al fallar un caso para que pudieran servir de modelo en casos similares. Más bien, la intención era educativa; de hecho, los Year Books no son, como se decía, law reports, sino compendios de las notas que abogados y estudiantes tomaban en el curso del pleading para su uso privado. Se trataba de preservar las lineas argumentales establecidas en el curso del pleading como recurso disponible para futuros practicantes, que aprendian así las estrategias y los movimientos a seguir para vencer al contrincante. En otras palabras, los Year Books eran repositorios a los que los abogados podian acudir para buscar los argumentos y las alegaciones más adecuadas para encauzar el pleading hacia la cuestión sobre la que se esperaba que el caso girara. El abogado medieval no tenía interés alguno en la formulación de principios abstractos de derecho substantivo; su preocupación primordial era desbancar a su oponente, y para ello necesitaba un conocimiento práctico acerca de los writs, del proceso y, sobre todo, de cómo conducirse en el pleading. ${ }^{14}$

El derecho medieval de Inglaterra no era un derecho jurisprudencial, sino un saber compartido y aceptado por los miembros de la profesión jurídica. Si algún valor tenía el precedente singular era como manifestación de ese saber común cuyos depositarios naturales, por supuesto, eran los jueces. Por eso, cuando en el curso del pleading se citaba un pleito se hacía de modo personal y no formal: se apelaba a la memoria del abogado o del juez que era invocada no con fuerza vinculante, sino como un ejemplo del problema en discusión. ${ }^{15}$

\section{EL PRECEDENTE EN LA ÉPOCA MODERNA}

$\mathrm{Si}$, como se ha dicho, la estructura del proceso medieval, así como el estilo de law reporting que derivaba del mismo, impedian el desarrollo de la doctrina del precedente vinculante en la edad media, los cambios que comenzaron a producirse en la estructura del proceso al final de este período inevitablemente dieron lugar a una nueva actitud con respecto al precedente.

En primer lugar, entre los siglos XV y XVI se abandonó el pleading oral y en su lugar se introdujo la práctica del pleading escrito. Puesto que esta modalidad no permitía la posibilidad de avanzar argumentos provisionales que luego podian ser emendados si no se vislumbraba su viabilidad, el pleading tentativo desapareció. Con la desaparición de mismo, la cuestión de hecho se podía ahora formular de manera más clara, y el foco de atención procesal podía trasladarse al momento de la sentencia. Igualmente, también se hizo posible distinguir claramente entre las cuestiones de hecho y las cuestiones de derecho, asegurándose que los law reports se centraran en las últimas. ${ }^{16}$

\footnotetext{
14 J. H. BAKER, An Introduction to English Legal History, op. cit., pp. 179-181, 197. T. F. T. PLUCKNETT, A Concise History of the Common law, op. cit., pp. 268-273, 345-347. T. E. LEWIS, "The History of Judicial Precedent. I", op. cit., 215-224.

15 J. H. BAKER, An Introduction to English Legal History, op. cit., 198. N. DUXBURY, The Nature and Authority of Precedent, op. cit., pp. 32-33.

16 J. H. BAKER, An Introduction to English Legal History, op. cit., pp. 81-82. T. F. T. PLUCKNETT, A Concise History of the Common law, op. cit., pp. 407-408.
} 
En segundo lugar, en los siglos modernos se desarrollaron las motions in banc, esto es, procedimientos que permitian plantear cuestiones de derecho tras el juicio. La más importante para el desarrollo de la doctrina del precedente fue la llamada motion in arrest of judgment, que cabe traducir como 'moción para detener la sentencia'. Se presentaba por el demandado (defendant) tras el veredicto, considerando que incluso después de demostrarse verdaderos los hechos alegados por el demandante, estos hechos no constituian una causa de acción. De esta manera, salvada la determinación de los hechos, se hacía posible formular cuestiones de derecho de modo efectivo, y no simplemente provisional, sin riesgo para la defensa. Y, por lo tanto, en consonancia con la introducción del pleading escrito, el foco se trasladaba de la fase previa al juicio que tenía como objeto producir la cuestión sometida al jurado, hacia la fase posterior al juicio, cuyo propósito era la emisión de una sentencia. Se puede ver así que estos cambios modificaron la función judicial. Si antes el juez se presentaba como un árbitro que trataba de guiar las partes hacia la cuestión de hecho, ahora el juez tenía que determinar el efecto jurídico de los hechos, una vez establecidos por el jurado. ${ }^{17} \mathrm{Y}$ para ello, puesto que las cuestiones de derecho se presentaban de manera firme, el juez tenía que producir decisiones razonadas, resolviéndolas no sólo para el caso en cuestión, sino también fijando precedentes para casos futuros. No en balde, a partir del siglo XVI, la actitud reticente de los jueces dio paso a una creciente suficiencia para fundamentar los fallos en los principios fijados en casos precedentes. ${ }^{18}$

Para que jueces y abogados invocaran los precedentes como fuentes normativas era condición necesaria que pudieran citarlos de forma cierta $\mathrm{y}$ precisa. El segundo elemento que jugó un papel fundamental en la fijación de la idea de precedente fue la invención de la imprenta. Gracias a este cambio tecnológico, fue posible la elaboración de law reports impresos que recogía eso casos en los que se discutían cuestiones de derecho después del veredicto. A diferencia de los Year Books medievales, esos nuevos law reports se centraron en las opiniones de jueces que luego serian citadas como razones para decidir en casos ulteriores.

Aunque es cierto que, debido a esos cambios, los precedentes comenzaron a verse como fuente normativa pues ofrecian las razones para decidir, también es cierto que la formulación de la doctrina del precedente vinculante estaba aún por llegar. De hecho, la teoría dominante en la época era todavía eco de la concepción medieval: los precedentes eran manifestación del derecho, pero no derecho ellos mismos. Se consideraba que el juez que determinaba una cuestión jurídica declaraba principios que existian desde tiempos inmemoriales, y por lo tanto parecía inconcebible que él mismo estableciera el derecho. Y, por supuesto, los jueces siempre tenían la libertad de considerar un precedente como expresión errónea de esos principios inmutables, procediendo entonces a declarar el principio que estimasen verdadero. En otras palabras, los tribunales no estaban obligados a seguir un precedente que consideraban incorrecto. Además, en la época moderna no existía una clara jerarquía en el sistema judicial y la doctrina del precedente vinculante dificilmente podia formularse sin ese criterio externo

\footnotetext{
17 J. H. BAKER, An Introduction to English Legal History, op. cit., pp. 82-85.

18 J. H. BAKER, An Introduction to English Legal History, op. cit., p. 82, pp. 198-99. N. DUXBURY , The Nature and Authority of Precedent, op. cit., pp. 33-34.
} 
que permite distinguir el valor de dos precedentes contradictorios entre sí. Tampoco se había desarrollado un método para distinguir las razones pertinentes al caso en cuestión de entre todo aquello que podia tener un valor persuasivo pero no vinculante, o de las diversas opiniones o votos particulares emitidos en el seno de órganos colegiados. 19

\section{LA DOCTRINA DEL PRECEDENTE EN EL SIGLO XIX}

Como indicaba al principio, fue a partir el siglo XVIII pero, sobre todo, desde mediados del XIX cuando se formuló la doctrina del precedente vinculante. De hecho, todavía en la década de 1830 no se había zanjado la cuestión de si, en aras de la certidumbre jurídica, la consistencia y la uniformidad en las sentencias, los jueces tenían que seguir los precedentes incluso si los consideraban erróneos; sólo en 1898 se pronunció la Cámara de los Lores al respecto, fijando la doctrina que ha dominado hasta mediados del siglo XX. ${ }^{20}$

Como razón que explicara por qué la doctrina del precedente vinculante cristalizó justo en ese momento se ha aducido que el law reporting comenzó a profesionalizarse a mediados del siglo XVIII. Igualmente, el desarrollo de la taquigrafia durante el primer tercio del siglo sucesivo permitió que los law reporters reprodujeran las opiniones judiciales de una manera fidedigna. Se hizo frecuente también remitir los reports a los mismos jueces para su corrección y aprobación antes de ser publicados. Todos estos factores contribuyeron a la precisión y completitud de los nuevos law reports y, por tanto, a que fuera cada vez más difícil ignorar o interpretar libremente las razones que los jueces daban para decidir. ${ }^{21}$

A estos factores materiales se suele unir la reforma judicial de 1873-1875, que organizó la jerarquía de los tribunales en Inglaterra. Con ella se estableció un criterio claro para distinguir entre precedentes contradictorios y se abrió paso la idea que las decisiones de un tribunal superior en jerarquía necesariamente vinculaban a los tribunales inferiores. Sin embargo, este factor no servía como criterio para decidir si los tribunales podian recusar sus propias decisiones. Y aunque la jerarquización de los órganos judiciales probablemente ayudó a consolidar una tendencia que estaba ahí, no se puede decir que fuese una condición suficiente, puesto es perfectamente concebible un sistema jurídico con tribunales jerarquizados y sin la doctrina del precedente vinculante. ${ }^{22}$

19 J. H. BAKER, An Introduction to English Legal History, op. cit., pp. 182-184. T. F. T. PLUCKNETT, A Concise History of the Common law, op. cit., pp. 348-349. T. E. LEWIS, "The History of Judicial Precedent. IV", LQ Rev., vol. 48 (1932), pp. 230-247. N. DUXBURY, The Nature and Authority of Precedent, op. cit., pp. 33-34. R. CROOS y H. J. W., Precedent in English Law, op. cit. pp. 27-30.

20 J. H. BAKER, An Introduction to English Legal History, op. cit., p. 199. T. F. T. PLUCKNETT, A Concise History of the Common law, op. cit., p. 350. R. CROOS y H. J. W. , Precedent in English Law, op. cit. p. 25.

21 J. H. BAKER, An Introduction to English Legal History, op. cit., p. 199. T. F. T. PLUCKNETT, A Concise History of the Common law, op. cit., p. 350. R. CROOS y H. J. W. , Precedent in English Law, op. cit. pp. 24-25.

22 J. H. BAKER, An Introduction to English Legal History, op. cit., pp. 199-201. T. F. T. PLUCKNETT, A Concise History of the Common law, op. cit., p. 350. R. CROOS y H. J. W. , Precedent in English Law, op. cit. p. 24-25. 
En el plano intelectual se ha sugerido por algunos autores que el factor decisivo fue, probablemente, la aparición de la escuela analítica del derecho, esto es, la variedad insular del positivismo jurídico que surgió con Jeremy Bentham y John Austin. Esta tesis sobre la influencia del positivismo en la doctrina del precedente vinculante se puede dividir en dos sub-tesis. En primer lugar, se ha argumentado que la filosofia positivista incluía el aparato conceptual que permitió a los abogados y jueces el escrutinio de los casos de manera nueva y transversal, más allá de las rígidas fronteras de las forms of action. De este modo, el positivismo habria contribuido a identificar los precedentes cada vez más como normas jurídicas, y no como testimonio de principios inmutables. En contra de esta tesis secundaria se ha esgrimido que supone una aseveración acerca de la influencia del positivismo sobre la ciencia juridica en general, siendo cuestionable y dificil de probar el impacto de las teorias positivistas sobre la judicatura. ${ }^{23}$

La segunda tesis secundaria, relacionada con la anterior, afirma que, puesto que la teoría positivista explicó la existencia de los precedentes y del propio common law como derecho creado por los jueces, cuando esta visión se convirtió en dominante fue más dificil invocar la teoría declaratoria del precedente para apartarse de casos previos. Como he afirmado, según esta doctrina declaratoria las decisiones no eran en sí mismas derecho sino prueba del derecho, una concepción que permitía al juez apartarse de decisiones previas siempre y cuando lo justificaran por tratarse de errores y declarasen el derecho correcto para la cuestión. Sin embargo, cuando el positivismo jurídico conceptualizó las decisiones jurídicas como mandatos tácitos de la soberanía y, por tanto, como derecho positivo, no fue posible sostener la teoría declaratoria. Al carecer entonces los jueces de bases para rechazar los precedentes que no quisieran seguir, no les quedó otro remedio que asumir su papel de legisladores. Ahora bien, como los jueces no estaban dispuestos a aceptar fácilmente ese papel se llegó inevitablemente al precedente vinculante. Contra esta tesis se ha argumentado que no se entiende que si la teoría positivista del precedente llevó a abandonar la teoría declaratoria, por qué la misma no condujo a los jueces a asumir abiertamente su papel creador. Además, como señalé en el punto anterior, no está claro cuál fue el impacto del positivismo sobre la judicatura inglesa. De hecho, y quizá este es el argumento decisivo, los tribunales continuaron esgrimiendo la teoría declaratoria del precedente durante gran parte del siglo XX. ${ }^{24}$

\section{PROSPECTIVAS DE ESTUDIO}

¿Significa eso que debemos abandonar la hipótesis de una conexión histórica entre el positivismo y la doctrina del precedente en Inglaterra? Con esto he llegado al verdadero motivo de estos apuntes, que no es otro que sugerir nuevas líneas de investigación. Un pasaje del famoso libro de John Henry Merryman sobre la tradición de civil law contiene un estímulo en tal sentido. Según Merryman la certidumbre jurídica se obtiene

\footnotetext{
23 M. LOBBAN, The Common law and English Jurisprudence, 1760-1850, Oxford University Press, Oxford, New York, 1991, pp. 235, 257-259, 288. N. DUXBURY, The Nature and Authority of Precedent, op. cit., pp. 37-39.
}

24 N. DUXBURY, The Nature and Authority of Precedent, op. cit., pp. 39-45. 
in the common law by giving the force of law to judicial decisions, something theoretically forbidden in civil law. The accumulation of judicial decisions in the course of time in a jurisdiction provides a variety of concrete, detailed examples of legal rules in operation. These, together with the statements of the rules themselves, are likely to provide more certainty about the law than are bare legislative statements of the rules. Thus, the desire for certainty is an argument in favor of stare decisis in the common law tradition, whereas it is an argument against stare decisis in the civil law tradition ${ }^{25}$.

Lo que llama la atención de este pasaje es su parecido a lo que escribió James Fitzjames Stephen, uno de los adalides de la codificación del common law en Inglaterra, al reflexionar sobre el problema de la certeza jurídica. ${ }^{26}$ La idea de fondo es que, lejos de lo que pudiera pensarse, un derecho jurisprudencial como el common law dejaba menos margen de maniobra a los jueces que cualquier código, por cuanto constituía un sistema de normas más completo al desarrollarse en el tiempo, caso a caso.

El problema de la certidumbre jurídica tiene que ver con una doctrina que casi siempre acompaña al positivismo en Inglaterra: me refiero a la separación de poderes. En el clásico esquema revolucionario, como sabemos, la producción de normas es competencia exclusiva de legislaturas representativas, mientras que los jueces se limitan a aplicar la ley. Por tanto, los jueces no pueden crear derecho y, en consecuencia, no pueden seguir decisiones judiciales previas. Sin embargo, en los países de common law ocurre lo contrario. ¿Por qué? La hipótesis con la que se puede abrir una nueva vía de investigación es que, durante la segunda mitad del XIX, el ascenso de los valores positivistas conllevó un cambio de actitud con respecto a los jueces y al common law. Puede que el positivismo como teoría no tuviera gran impacto en la judicatura, pero no puede negarse que el ideal de la certeza en el derecho y de la separación de poderes se convirtiera en un lugar común del pensamiento político y jurídico de la Inglaterra victoriana. En otras palabras, quizá la aparición de la doctrina del precedente vinculante pueda explicarse como resultado de la reconceptualización del common law y de papel del juez desde el punto de vista positivista. Ahora bien, para entender esa reconceptualización debemos comprender qué tipo de legislador es el juez dentro del modelo positivista. El juez colma lagunas en el ordenamiento, legisla allí donde el legislador no ha hablado. Una vez que el juez aparece como legislador, aunque sea de manera imperfecta y particular, y que el common law aparece como una acumulación de normas particulares, es fácil dar un paso más y pensar que con el paso del tiempo los jueces inevitablemente estarán vinculados por sus propias normas. Es decir, esta es una manera de justificar la existencia del common law frente a aquellos que argumenten que bajo la teoria declaratoria del derecho se esconden la arbitrariedad y la incertidumbre jurídica.

25 J. H. MERRYMAN, The Civil Law Tradition: An Introduction to the Legal Systems of Western Europe and Latin America, $2^{\mathrm{a}}$ ed., Stanford University Press, Stanford, California, 1985, pp. 48-49.

26 J. F. STEPHEN, A History of the Criminal Law of England, vol. 3, MacMillan and Co., London, 1883, pp. 352-3 


\section{BIBLIOGRAFÍA}

J. H. Baker, An Introduction to English Legal History, $4^{\mathrm{a}}$ ed., Londres, Dayton (Ohio), Butterworths, LexisNexis, 2002.

R. Cross, y H, J. W., Precedent in English Law. 4 ${ }^{\mathrm{a}}$ ed., Oxford University Press, Oxford, Nueva York, 1991.

N. Duxbury, The Nature and Authority of Precedent, Cambridge University Press, Cambridge, 2008.

A. L. Goodhart, "Precedent in English and Continental Law", L. Q. Rev., vol. 50, 1934, pp. 40-65.

A. L. Goodhart, "Case Law-A Short Replication”, L. Q. Rev., vol. 50, 1934, pp. 196200.

A. L. Goodhart, "Determining the Ratio Decidendi of a Case", The Yale Law Journal, vol. 40, num. 2, 1930, pp. 161-183.

T. E. Lewis, "The History of Judicial Precedent. I", L. Q. Rev., vol. 46, 1930, pp. 207-224.

T. E, Lewis “The History of Judicial Precedent. II", L. Q. Rev., vol. 46, 1930, pp. 341-360.

T. E. Lewis, "The History of Judicial Precedent. III". L. Q. Rev., vol. 47, 1931, pp. 411-427.

T. E. Lewis, "The History of Judicial Precedent. IV", L. Q. Rev., vol. 48, 1932, pp. 230-247.

M. Lobban, The Common law and English Jurisprudence, 1760-1850, Oxford, Oxford University Press, Nueva York, 1991.

J. H. Merryman, The Civil Law Tradition: An Introduction to the Legal Systems of Western Europe and Latin America, $2^{\mathrm{a}}$ ed., Stanford University Press, Stanford, Calif., 1985.

T. F, T. Plucknett, A Concise History of the Common law., $5^{\mathrm{a}}$ ed., Little, Brown and Co., Boston, 1956.

J. F., Stephen A History of the Criminal Law of England, vol. 3, MacMillan and Co., Londres, 1883.

Enviado el (Submission Date): 05/09/2018

Aceptado el (Acceptance Date): 23/11/2018 